\title{
La actividad científica en ciencias de la UCLM durante el período 2000-2004
}

The scientific activity at the University of Castilla-La Mancha, from 2000 to 2004

\author{
María Luisa Pérez Aliende \\ UCLM. Biblioteca General de Cuenca, Avda. de los Alfares 42, Cuenca, mluisa.perez@uclm.es
}

\begin{abstract}
Resumen
El objetivo principal de este estudio es conocer, a través de sus publicaciones, la actividad científica de los investigadores de la Universidad de Castilla-La Mancha en ciencias puras, experimentales y tecnológicas, durante el período comprendido entre los años 2000 y 2004. Las fuentes de información utilizadas son las bases de datos del ISI accesibles a través de la plataforma Web of knowledge. El tipo documental analizado es el artículo de revista y la clasificación temática empleada es la de las bases de datos del ISI. Los resultados permiten conocer aspectos relacionados con la producción, calidad y repercusión de la ciencia, a partir del análisis de las distribuciones, que determinarán el idioma utilizado por los investigadores para publicar sus resultados de investigación, la temática de los artículos analizados a partir de las citas recibidas, la visibilidad internacional de los trabajos en función de las revistas en las que se han publicado los artículos, del factor de impacto y la asignación por cuartiles, así como del análisis de las citas recibidas.
\end{abstract}

Palabras clave: Actividad científica. Visibilidad de la información. Análisis de citas. Revistas científicas.

\section{Introducción}

Los estudios bibliométricos aplicados a la actividad científica de una institución proporcionan una visión general de la investigación que está desarrollando el personal que trabaja en la misma. Existen muchos trabajos que analizan la producción científica en España como un conjunto, pero pocos son los que se centran en una institución en particular como puede ser la universidad (Moya, 2005). Las publicaciones científicas son el principal medio de difusión de la ciencia que genera una institución (Pérez, 1999).

La universidad desempeña un papel básico en la investigación española y de hecho es el principal sector institucional productor de publicaciones científicas (Bordons y Gómez, 1997).

\begin{abstract}
The main goal of this study is get to know the scientific research done at the University of Castilla-La Mancha in pure, experimental, and technological sciences, during 2000 and 2004. The main sources of information have been the ISI data bases; the article is the type of document analyzed; and the ISI subject classification has been used.

The results report data on productivity output, quality, reliability, language and main topics, and the international visibility and impact of its papers depending on the journals in which they have been published, measuring the impact factor and quartiles as a tool for ranking and categorizing journals, and the citations received by other authors.
\end{abstract}

Keywords: Scientific activity. Information visibility. Citation analysis. Scientific journals.

El Plan Regional de Investigación Científica, Desarrollo Tecnológico e Innovación (PRINCET) en 2005 concluye que en la Comunidad Autónoma de Castilla-La Mancha, el entorno científico está básicamente integrado por la Universidad de Castilla-La Mancha (en adelante UCLM), con más de 2000 profesores, 36 facultades 0 escuelas y unos 32.000 alumnos. Representa el $42 \%$ del gasto en $1+D+i$ de la Comunidad y el $44,5 \%$ del personal que se dedica a estas actividades. Aporta al sistema regional el $81 \%$ de los investigadores del entorno científico.

Hay que tener en cuenta que los hábitos de publicación de los investigadores son diferentes: mientras que en la ciencia básica, la ciencia internacional, los resultados se transmiten a través de revistas internacionales, la ciencia aplicada encuentra su medio de difusión en las revistas de ámbito nacional. Este hecho queda 
patente en las ciencias sociales y humanidades en las que se realiza una investigación de carácter local, tanto por el alcance de los temas como por los factores lingüísticos o culturales. Por ello, afectan a un colectivo menor, aunque no por ello dejan de ser importantes para el desarrollo de la actividad investigadora de un país (Gómez y Bordons, 1996).

Desde hace unos años existe una gran preocupación por parte de los investigadores por publicar en revistas de ámbito internacional, sobre todo aquellas que recoge el Institute for Scientifc Information (ISI) en sus bases de datos y que aportan el factor de impacto de cada revista para un año determinado, dato no disponible para las revistas de ámbito nacional por no existir una herramienta que aporte unos datos similares. Las repercusiones son económicas, pues les permite beneficiarse de los incentivos por los tramos de investigación (sexenios), según está regulado de forma explícita en las medidas legales adoptadas por el gobierno en lo relativo a la evaluación de los investigadores españoles.

\section{Objetivos}

El objetivo que se pretende con este estudio es conocer la actividad científica de los investigadores de la UCLM durante el período 2000 al 2004 y la visibilidad internacional de sus trabajos a partir de las revistas en las que se han publicado y las citas que han recibido por sus colegas. Todo ello recogido en las bases de datos del ISI. Estas bases de datos son un instrumento muy utilizado en los estudios bibliométricos por su carácter multidisciplinar y la difusión internacional de las revistas seleccionadas con criterios de calidad y alcance internacional.

Los objetivos específicos que se persiguen están relacionados con la producción, la calidad y la repercusión de la ciencia y son:

- Conocer el idioma de publicación de los artículos realizados por el personal docente e investigador de la UCLM.

- Determinar las temáticas de su actividad científica.

- Estudiar la visibilidad internacional del conocimiento que se ha generado.

- Analizar las citas que han recibido los artículos publicados.

\section{Metodología}

Por actividad científica se entiende en este trabajo el conjunto de artículos publicados por el personal docente e investigador de la UCLM entre los años 2000-2004.

Las fuentes de información para este estudio son las bases de datos del ISI, Web of Science: Science Citation Index (SCI), Social Science Citation Index (SSCl) y Arts and Humanities Citation Index (A\&HCl); y los Journal Citation Reports (JCR), accesibles a través de la plataforma Web of knowledge. Son bases de datos muy utilizadas a la hora de realizar estudios bibliométricos por ser de tipo multidisciplinar y por su carácter internacional. Cuentan con ventajas y limitaciones. Entre las ventajas, destaca el hecho de que el SCl es la base de datos más utilizada para analizar la difusión internacional de la literatura científica (Moya, 2005). Entre los inconvenientes, se pueden resaltar los sesgos lingüístico, geográfico y disciplinar a favor de la ciencia básica, las ciencias médicas y experimentales frente a la aplicada y a las ciencias sociales (Bordons y Gómez, 1997).

La búsqueda se ha realizado a partir de la afiliación de los autores, utilizando la estrategia de búsqueda, en el campo address, "((univ castilla la mancha) or uclm)", recuperando únicamente los artículos publicados entre los años 2000 y 2004, ambos inclusive, en las tres bases de datos — $\mathrm{SCl}, \mathrm{SSCl}$ y $\mathrm{A} \& \mathrm{HCl}$ - , considerando los resultados de estas dos últimas sólo para el computo del número de artículos indizados y para conocer el idioma de publicación. El campo address recoge los lugares de trabajo de los autores y es fundamental para conocer la producción científica de una institución -en este caso de la UCLM- y la colaboración científica (que no se va a abordar en el presente estudio). Para el tratamiento automatizado de los datos se transfirieron los registros recuperados (1394) a una base de datos en Procite 5.0, que permitirá efectuar las búsquedas pertinentes y recuperar los ítems para su tratamiento. De esta base de datos los campos de los que se ha obtenido la mayoría de la información han sido el campo de notas, idioma y el de título de las publicaciones. Los resultados de estas búsquedas se tabularán en una hoja de cálculo en Excel 2000 para el análisis posterior.

El tipo documental analizado es el artículo de revista. La clasificación temática empleada es la de las bases de datos del ISI, que asigna las revistas entre más de 150 materias pudiendo aparecer cada título hasta en cinco diferentes. La adscripción de los documentos a una materia científica se realizará de acuerdo a la información de la revista y la temática recogida en la base de datos Journal Citation Reports (JCR) del ISI correspondiente al año 2004. Las materias se han obtenido directamente a través del 
análisis y asignación efectuado por las propias bases de datos del ISI a partir de los resultados de la búsqueda de los documentos producidos por la UCLM. Se han transferido los resultados desde la página del Web of Science a un fichero en Excel para su tratamiento posterior. En este apartado se ha empleado el método del recuento total, que asigna cada documento completo a todas las materias que lo describen, lo que produce duplicados a la hora de los recuentos y que presenta el inconveniente de que la suma total sea superior al de documentos estudiados (Gómez et al., 2003).

El factor de impacto $(\mathrm{Fl})$ será el indicador utilizado para conocer la visibilidad internacional y la difusión de los resultados de la investigación. Por FI referido a una revista científica se entiende la tasa de citas bibliográficas obtenidas por artículos publicados resultante de dividir la cantidad de citas recibidas cada año por la revista entre el número total de estos mismos artículos (Ferreiro, 1993).

\section{Resultados}

Durante el período objeto de estudio (20002004) tal como se expone en la Tabla I, la UCLM produjo 1394 artículos con visibilidad internacional, 1354 procedentes del área de las ciencias, 20 de las ciencias sociales y 20 del arte y las humanidades.

\begin{tabular}{lrrrrrr}
\hline Bdd. & 2000 & 2001 & 2002 & 2003 & 2004 & Total \\
\hline $\mathrm{SCl}$ & 192 & 212 & 283 & 333 & 334 & 1354 \\
\hline $\mathrm{SSCl}$ & 4 & 3 & 5 & 2 & 6 & 20 \\
\hline $\mathrm{A} \& \mathrm{HCl}$ & 2 & 2 & 4 & 6 & 6 & 20 \\
\hline Total & 198 & 217 & 292 & 341 & 346 & 1394 \\
\hline
\end{tabular}

Tabla I. Artículos de la UCLM indizados en las bases de datos del ISI.

El número de artículos analizados anualmente por el ISI es poco significativo tanto para el caso del SSCI $(1,43 \%)$ como para el $\mathrm{A} \& \mathrm{HCl}(1,43 \%)$, por lo que queda de manifiesto la presencia prácticamente nula en estas bases de datos de la actividad de la UCLM.

\subsection{Idioma de publicación de los artículos}

De los 1394 artículos, los publicados en lengua inglesa constituyen el $97,6 \%$ del total de los estudiados. El inglés es el idioma dominante en el $96,1 \%$ de los artículos del SCl, y constituyen el $98,45 \%$ del total de los publicados en esta lengua, y el $90 \%$ de los del SSCl, si bien la presencia de estos últimos es muy escasa. El es- pañol es el idioma de publicación en el $75 \%$ de los artículos del $\mathrm{AHCl}$. Todos los artículos en español se publicaron en revistas nacionales incluidas en los índices del ISI.

\begin{tabular}{lrrrr}
\hline & $S C l$ & $S S C l$ & $A H C l$ & Total \\
\hline Inglés & 1339 & 18 & 3 & 1360 \\
\hline Español & 14 & 2 & 15 & 31 \\
\hline Francés & 1 & 0 & 1 & 2 \\
\hline Italiano & 0 & 0 & 1 & 1 \\
\hline Total & 1354 & 20 & 20 & 1394 \\
\hline
\end{tabular}

Tabla II. Idioma de los artículos.

\subsection{Temática de los artículos}

Se ha estudiado la temática de los 1354 artículos publicados en revistas analizadas por el SCl. Siguiendo la clasificación temática de las revistas del ISI se han estudiado 55 materias para los artículos contenidos en el SCl.

Las materias científicas con mayor presencia en la Universidad (Tabla III, en apéndice) en función del número de citas recibidas son: química orgánica (670 citas), química inorgánica y nuclear (556), bioquímica y biología molecular (479), química física (438) y neurociencias (402). De las que más artículos se han producido son tecnología de los alimentos (89 ítems), química analítica (87), química orgánica (79), química física (78) y química inorgánica y nuclear (77); todas ellas han superado los 70 artículos.

Si nos centramos en la ratio de citas por artículo para cada materia, sólo tres alcanzan valores superiores a 10: biología celular $(12,38)$, electroquímica (11) y óptica (11), son materias con poca actividad científica, 13, 14 artículos durante los cuatro años, pero con alta citación posterior por parte de otros investigadores. Si este valor desciende hasta 7 , la selección se amplia a endocrinología y metabolismo $(9,85)$, física nuclear y química $(9,72)$, biofísica $(9,43)$, bioquímica y biología molecular $(9,21)$, física $(8,83)$, ingeniería medioambiental $(8,6)$, química orgánica $(8,48)$ y química inorgánica y nuclear $(7,22)$. De este modo se puede concluir que tanto química orgánica como inorgánica y nuclear son las materias más productivas y que a su vez tienen una mayor citación.

Las materias con una ratio de citas por artículo menor, e inferior a 2 , han sido respectivamente, estadística y probabilidad $(1,4)$, ingeniería metalúrgica $(1,56)$, investigación operativa $(1,69)$ y matemáticas $(1,95)$. Que a su vez son las materias que menos citas han recibido por sus traba- 
jos, con 14 en 10 trabajos, 25 en 16 artículos, 27 en 16 y, por último, 43 en 22 artículos, respectivamente.

Existen 78 materias más que no se han incluido en este cuadro por tener una producción inferior a los 10 artículos entre los años 2000-2004.

Teniendo en cuenta el número de artículos publicados en una temática y el número de citas que recibieron para cada año estudiado (Tabla IV, en apéndice), se obtuvieron los siguientes datos. En el año 2004 ninguna materia superó las 80 citas, hecho que puede tener dos lecturas diferentes: por un lado, un descenso en la calidad de los trabajos, o, por contrario, son artículos que se han publicado recientemente y que quizás en unos años la citación que reciban sea mayor. De 2000 a 2003 son química orgánica (con 181, 219, 133 y 92 citas respectivamente), química inorgánica y nuclear $(159,126,117$ y 93) y química física $(141,102,117$ y 57) las más citadas anualmente. Para los trabajos publicados en el 2003 tecnología de los alimentos (96) y neurociencias (89) son las otras disciplinas más citadas; en el 2002 son neurociencias (148), una vez más, ingeniería eléctrica y electrónica (125) y bioquímica y biología molecular (104); en el 2001, bioquímica y biología molecular (174), ecología (120), ciencias medioambientales (102), óptica (99), neurociencias (89) y biología celular (95), y en el 2000 física nuclear, molecular y química (121), tecnología de los alimentos (103), química analítica (102), física aplicada (97), bioquímica (94) e ingeniería eléctrica y electrónica (80).

2001 es el año en que se publican los trabajos que más se han citado en trabajos posteriores y 2004 el que menos.

En el año 2004, de las 55 materias que se han estudiado, en 23 no se ha superado el número de 10 citas; en el 2003 y en el año 2000, ocurre en 16; en el 2002 en 17; y en el año 2001 sólo en 7 .

No se produjo ningún artículo durante el año 2000 de parasitología; física, condensación de materias; estadística y probabilidad; y electroquímica. En ingeniería metalúrgica se publicaron 2 artículos que no recibieron ninguna cita posterior. En el 2001 no hubo producción en espectroscopia; farmacología y farmacia; informática, aplicaciones; ingeniería mecánica; e investigación operativa. En el año 2002 se publicaron trabajos sobre todas las materias, si bien no recibió ninguna cita estadística y probabilidad. Esta misma materia es la única que en el año 2003 no tiene producción. Por último en el 2004 se dan a conocer estudios en todas las materias, pero los de biofísica y matemáticas no reciben ninguna cita en trabajos posteriores.

\subsection{Visibilidad de la producción}

Todos los trabajos analizados en el $\mathrm{SCl}$ se publicaron en 620 revistas; las analizadas (21) recogen el $21 \%$ de los ítems estudiados en el período.

Como se ha indicado anteriormente desde hace unos años existe una gran preocupación por parte de los docentes e investigadores por publicar en revistas de ámbito internacional, sobre todo aquellas que recoge el ISI en sus bases de datos y que aportan el FI de cada revista para un año determinado.

Del JCR se han tomado las materias estudiadas y FI correspondiente a cada título de revista que permite medir la influencia en la comunidad científica. Hay que tener en cuenta unas limitaciones al uso del FI, como puede ser el hecho que no se pueden efectuar comparaciones entre las categorías temáticas, pues se ve influido por el tamaño de la comunidad científica, de sus hábitos de publicación y del carácter básico o aplicado de la investigación. La investigación aplicada suele utilizar una bibliografía menos reciente que la básica, de ahí que sea necesario manejar por separado el FI de cada materia, tal como se expone en el estudio publicado en 2003 sobre la Región de Murcia (Gómez et al., 2003).

Para realizar el estudio, todos los títulos de las publicaciones científicas recogidos en el JCR de 2004 para cada categoría temática se han ordenado por FI. Este hecho ha permitido determinar la posición que ocupan en el JCR los títulos utilizados por los investigadores de la UCLM y el cuartil en el que se encuentran.

En la siguiente tabla se recogen los 21 títulos de las revistas en las que se han publicado la mayoría de los artículos, es decir, las preferidas por los investigadores de la UCLM para difundir los resultados de sus investigaciones, así como su posición en el JCR de 2004. De ellas 13 se sitúan en el primer cuartil de su categoría, 6 en el segundo, una en el tercero y una en el cuarto. En su mayoría pertenecen al área de la química, de repercusión internacional.

Los títulos de las revistas seleccionadas difunden artículos que por lo menos han recibido 20 citas. En la Tabla VI (en apéndice) se presentan las revistas en las que se han publicado los artículos más citados de los analizados en el $\mathrm{SCl}$. Se indica el factor de impacto de la publicación según los datos obtenidos en el JCR del 2004 y la posición que ocupa de acuerdo al FI 
entre las revistas de su categoría, lo que nos permite conocer el cuartil en el que se encuentra. En esta situación se han estudiado 36 revistas $(5,69 \%)$ que han publicado 51 artículos $(3,76 \%)$ y que, a su vez, han recibido 1417 citas, es decir, el $21,68 \%$ de todas las citas recibidas durante ese período. Predominan los títulos de química orgánica, e inorgánica, bioquímica, neurociencias y electroquímica.

\begin{tabular}{lrrrr}
\hline \multicolumn{1}{c}{ Título } & $\begin{array}{r}N^{\circ} \\
\text { Art. }\end{array}$ & $\begin{array}{c}N^{\circ} \\
\text { Cit. }\end{array}$ & $\begin{array}{r}\text { Pos. } \\
\text { JCR }\end{array}$ & Cuartil \\
\hline IEEE Trans. Power Syst & 33 & 255 & $84 / 209$ & 2 \\
\hline J. Organomet. Chem. & 23 & 125 & $17 / 45$ & 2 \\
\hline Anal. Chim. Acta & 19 & 92 & $12 / 70$ & 1 \\
\hline Ind. Eng. Chem. Res. & 18 & 89 & $17 / 116$ & 1 \\
\hline Chem. Phys. Lett. & 17 & 127 & $29 / 108$ & 2 \\
\hline J. Agric. Food Chem. & 16 & 105 & $1 / 29$ & 1 \\
\hline Organometallics & 16 & 191 & $8 / 45$ & 1 \\
\hline Phys. Rev. E & 15 & 105 & $3 / 21$ & 1 \\
\hline Eur. J. Inorg. Chem. & 13 & 43 & $12 / 45$ & 2 \\
\hline J. Phys. Chem. A & 13 & 79 & $27 / 108$ & 1 \\
\hline Phys. Rev. B & 13 & 53 & $8 / 60$ & 1 \\
\hline Chromatographia & 12 & 40 & $42 / 70$ & 3 \\
\hline Appl. Catal. A-Gen. & 10 & 62 & $31 / 108$ & 2 \\
\hline J. Org. Chem. & 10 & 124 & $7 / 58$ & 1 \\
\hline Lect. Notes Comput. Sc. & 9 & 7 & $53 / 70$ & 4 \\
\hline Agric. Water Manage. & 8 & 37 & $22 / 50$ & 2 \\
\hline Food Chem. & 8 & 41 & $14 / 58$ & 1 \\
\hline J. Appl. Phys. & 8 & 23 & $12 / 79$ & 1 \\
\hline Dalton Trans. & 8 & 68 & $9 / 45$ & 1 \\
\hline Phys. Rev. A & 8 & 112 & $7 / 54$ & 1 \\
\hline Talanta & 8 & 28 & $13 / 70$ & 1 \\
\hline Total: & 1806 & & \\
\hline & & & & \\
\hline
\end{tabular}

Tabla V. Revistas más utilizadas por los investigadores de la UCLM.

Se observa que 27 publicaciones están en el primer cuartil de su categoría temática, 7 en el segundo, y una respectivamente en el tercer y cuarto cuartil. Por lo que los artículos más citados se han publicado en las revistas de mayor visibilidad internacional.

Con la idea de mostrar la categoría de las revistas preferidas por los investigadores de la UCLM para difundir los resultados de sus estudios tal como hicieron Licea de Arenas junto con otros autores en el trabajo publicado en 2005, y sin olvidar que cada materia tiene un comportamiento diferente y no se pueden mezclar, se han agrupado los artículos más citados en función del FI de las revistas en que se publicaron (Tabla VII). Sólo cinco artículos se han publica- do en revistas con un factor de impacto superior a siete y han recibido el $3,38 \%$ de todas las citaciones del campo de las ciencias. El mayor número de artículos, 30 se encuentra en publicaciones periódicas con un FI que oscila entre 2 y 4 y representa el $11,6 \%$ del total de las citas.

\begin{tabular}{llrlr}
\hline & & $\%$ & & $\%$ \\
F.I. & $N^{\circ}$ art. & 1354 & $N^{\circ}$ citas & 6537 \\
\hline $7<\mathrm{FI}$ & 5 & $0,37 \%$ & 221 & $3,38 \%$ \\
\hline $4<\mathrm{FI}<7$ & 5 & $0,37 \%$ & 161 & $2,46 \%$ \\
\hline $2<\mathrm{FI}<4$ & 30 & $2,22 \%$ & 762 & $11,66 \%$ \\
\hline $0<\mathrm{FI}<2$ & 11 & $0,81 \%$ & 273 & $4,18 \%$ \\
\hline Total: & 51 & $3,77 \%$ & 1417 & $21,68 \%$ \\
\hline
\end{tabular}

Tabla VII. Factor de impacto de las revistas fuente de los artículos más citados en el SCI.

\subsection{Análisis de las citas recibidas}

De los 1354 artículos analizados se han citado 1069 (78,95\%) generando un total de 6537 citas. Se observa un incremento en el número de artículos indizados del $\mathrm{SCl}$ durante el período objeto de estudio en un $42,51 \%$. De esta manera, se paso de analizar 192 artículos en el año 2000 a 334 en el 2004, estabilizándose la productividad en los dos últimos años (2003 y 2004); hecho que contrasta con la citación que reciben en otros documentos. Según se expone en la Tabla VIII, en el año 2000 se publicaron el $14,18 \%$ del total de los artículos, que recibieron el $22,64 \%$ del total de citas. En el 2004 se publican el $24,67 \%$ de los artículos, mientras que se citan el del $18,80 \%$ del total. Respectivamente, el 2003 es el año con mayor porcentaje de artículos citados $(23,95 \%)$, y los artículos publicados en 2001 son los que más citas reciben $(24,35 \%)$ del total.

\begin{tabular}{lllllll}
\hline Años & Art. & $\%$ & $\begin{array}{l}\text { Art. } \\
\text { Cit. }\end{array}$ & $\%$ & Citas & $\%$ \\
\hline 2000 & 192 & $14,18 \%$ & 168 & $15,72 \%$ & 1480 & $22,64 \%$ \\
\hline 2001 & 212 & $15,66 \%$ & 193 & $18,05 \%$ & 1592 & $24,35 \%$ \\
\hline 2002 & 283 & $20,90 \%$ & 251 & $23,48 \%$ & 1567 & $23,97 \%$ \\
\hline 2003 & 333 & $24,59 \%$ & 256 & $23,95 \%$ & 1163 & $17,79 \%$ \\
\hline 2004 & 334 & $24,67 \%$ & 201 & $18,80 \%$ & 735 & $11,24 \%$ \\
\hline Total & 1354 & $100 \%$ & 1069 & $100 \%$ & 6537 & $100 \%$ \\
\hline
\end{tabular}

Tabla VIII. Presencia de la UCLM en el SCI.

El $87,5 \%$ de los artículos publicados en el año 2000 fueron citados, el $91,04 \%$ de los producidos en el 2001 (15,66\% del total), $88,69 \%$ de los 
del 2002, año en el que se publicaron el $20,90 \%$ de los artículos analizados, $76,88 \%$ de los del 2003 y $60,18 \%$ de los del 2004. De este modo se puede indicar que, a medida que la actividad científica reflejada en forma de artículo se ha ido incrementando, el interés para otros investigadores y la citación ha ido descendiendo. El 79\% de los artículos analizados en el $\mathrm{SCl}$ fueron citados en alguna ocasión.

En las siguientes tablas (IX-XIII) se ha recogido la distribución de citas del $\mathrm{SCl}$ por artículos en cada año objeto de estudio. Cada tabla está compuesta por cinco columnas con referencias al número de citas que ha recibido un trabajo, seguido del número de artículos que han recibido esa cantidad, en número total de citas, el tanto por ciento de artículos que contienen esa cantidad de citas y el tanto por cierto de citas recibidas.

\begin{tabular}{|c|c|c|c|c|}
\hline \multicolumn{5}{|c|}{ Articulos citados 2004} \\
\hline Citas/Art. & $N^{\circ}$ art. & $N^{\circ}$ citas & $\%$ Art. & $\%$ Citas \\
\hline 22 & 1 & 22 & $0,30 \%$ & $2,99 \%$ \\
\hline 20 & 1 & 20 & $0,30 \%$ & $2,72 \%$ \\
\hline 17 & 1 & 17 & $0,30 \%$ & $2,31 \%$ \\
\hline 15 & 1 & 15 & $0,30 \%$ & $2,04 \%$ \\
\hline 14 & 1 & 14 & $0,30 \%$ & $1,90 \%$ \\
\hline 13 & 3 & 39 & $0,90 \%$ & $5,31 \%$ \\
\hline 12 & 2 & 24 & $0,60 \%$ & $3,27 \%$ \\
\hline 11 & 1 & 11 & $0,30 \%$ & $1,50 \%$ \\
\hline 10 & 1 & 10 & $0,30 \%$ & $1,36 \%$ \\
\hline 9 & 11 & 99 & $3,29 \%$ & $13,47 \%$ \\
\hline 8 & 1 & 8 & $0,30 \%$ & $1,09 \%$ \\
\hline 7 & 6 & 42 & $1,80 \%$ & $5,71 \%$ \\
\hline 6 & 8 & 48 & $2,40 \%$ & $6,53 \%$ \\
\hline 5 & 14 & 70 & $4,19 \%$ & $9,52 \%$ \\
\hline 4 & 18 & 72 & $5,39 \%$ & $9,80 \%$ \\
\hline 3 & 22 & 66 & $6,59 \%$ & $8,98 \%$ \\
\hline 2 & 49 & 98 & $14,67 \%$ & $13,33 \%$ \\
\hline 1 & 60 & 60 & $17,96 \%$ & $8,16 \%$ \\
\hline 0 & 133 & 0 & $39,82 \%$ & $0,00 \%$ \\
\hline Total: & 334 & 735 & $100,00 \%$ & $100,00 \%$ \\
\hline
\end{tabular}

Tabla IX. Citas recibidas: artículos publicados en 2004.

Como puede observarse en las Tablas IX y X, durante los años 2004 y 2003 sólo hay un trabajo que ha sido mencionado en más de 30 ocasiones; en el año 2002, (Tabla XI) tres trabajos se citaron en 35,33 y 30 ocasiones respectivamente; en el año 2001 (Tabla XII), se publicó el documento que ha sido mencionado en más ocasiones durante los años estudiados, hasta recibir un total de 86 citas, otros cuatro artículos más superaron las 30; cantidad alcanzada en cinco ocasiones en el año 2000 (Tabla XIII). En este año, se publicó el segundo artículo más citado con un total de 58 . El 39,82\% los trabajos que se analizan publicados en el 2004 no han sido citados en ninguna ocasión; en el 2003 esta cifra alcanza el valor de un $23 \% ; 11 \%$ en el año 2002; en el 2000, 12,5\%; y es en el 2001 cuando esta cifra es más baja con un $9 \%$. En resumen, durante el año 2001 se publica el artículo más utilizado en trabajos posteriores y es cuando menos trabajos no se citaron por parte de otros investigadores.

\begin{tabular}{lrrrr}
\hline $\begin{array}{l}\text { Articulos citados } 2003 \\
\text { Citas/Art. }\end{array}$ & $N^{0}$ art. & $N^{\circ}$ citas & $\%$ Art. & $\%$ Citas \\
\hline 30 & 1 & 30 & $0,30 \%$ & $2,58 \%$ \\
\hline 25 & 1 & 25 & $0,30 \%$ & $2,15 \%$ \\
\hline 24 & 1 & 24 & $0,30 \%$ & $2,06 \%$ \\
\hline 22 & 1 & 22 & $0,30 \%$ & $1,89 \%$ \\
\hline 18 & 1 & 18 & $0,30 \%$ & $1,55 \%$ \\
\hline 17 & 2 & 34 & $0,60 \%$ & $2,92 \%$ \\
\hline 15 & 3 & 45 & $0,90 \%$ & $3,87 \%$ \\
\hline 14 & 2 & 28 & $0,60 \%$ & $2,41 \%$ \\
\hline 13 & 1 & 13 & $0,30 \%$ & $1,12 \%$ \\
\hline 12 & 7 & 84 & $2,10 \%$ & $7,22 \%$ \\
\hline 11 & 4 & 44 & $1,20 \%$ & $3,78 \%$ \\
\hline 10 & 1 & 10 & $0,30 \%$ & $0,86 \%$ \\
\hline 9 & 4 & 36 & $1,20 \%$ & $3,10 \%$ \\
\hline 8 & 12 & 96 & $3,60 \%$ & $8,25 \%$ \\
\hline 7 & 16 & 112 & $4,80 \%$ & $9,63 \%$ \\
\hline 6 & 15 & 90 & $4,50 \%$ & $7,74 \%$ \\
\hline 5 & 19 & 95 & $5,71 \%$ & $8,17 \%$ \\
\hline 4 & 29 & 116 & $8,71 \%$ & $9,97 \%$ \\
\hline 3 & 32 & 96 & $9,61 \%$ & $8,25 \%$ \\
\hline 2 & 41 & 82 & $12,31 \%$ & $7,05 \%$ \\
\hline 1 & 63 & 63 & $18,92 \%$ & $5,42 \%$ \\
\hline 0 & 77 & 0 & $23,12 \%$ & $0,00 \%$ \\
\hline Total: & 333 & 1163 & $100 \%$ & $100 \%$ \\
\hline & & & & \\
\hline
\end{tabular}

Tabla X. Citas recibidas: artículos publicados en 2003.

\begin{tabular}{|c|c|c|c|c|}
\hline \multicolumn{5}{|c|}{ Articulos citados 2002} \\
\hline Citas/Art. & $N^{\circ}$ art. & $N^{\circ}$ citas & $\%$ Art. & $\%$ Citas \\
\hline 35 & 1 & 35 & $0,35 \%$ & $2,23 \%$ \\
\hline 33 & 1 & 33 & $0,35 \%$ & $2,11 \%$ \\
\hline 30 & 1 & 30 & $0,35 \%$ & $1,91 \%$ \\
\hline 26 & 1 & 26 & $0,35 \%$ & $1,66 \%$ \\
\hline 25 & 1 & 25 & $0,35 \%$ & $1,60 \%$ \\
\hline 24 & 2 & 48 & $0,71 \%$ & $3,06 \%$ \\
\hline 23 & 1 & 23 & $0,35 \%$ & $1,47 \%$ \\
\hline 21 & 1 & 21 & $0,35 \%$ & $1,34 \%$ \\
\hline 20 & 3 & 60 & $1,06 \%$ & $3,83 \%$ \\
\hline 19 & 4 & 76 & $1,41 \%$ & $4,85 \%$ \\
\hline 18 & 4 & 72 & $1,41 \%$ & $4,59 \%$ \\
\hline 17 & 1 & 17 & $0,35 \%$ & $1,08 \%$ \\
\hline 16 & 1 & 16 & $0,35 \%$ & $1,02 \%$ \\
\hline 14 & 4 & 56 & $1,41 \%$ & $3,57 \%$ \\
\hline
\end{tabular}




\begin{tabular}{|c|c|c|c|c|}
\hline \multicolumn{5}{|c|}{ Articulos citados 2002} \\
\hline Citas/Art. & $N^{\circ}$ art. & $N^{\circ}$ citas & $\%$ Art. & $\%$ Citas \\
\hline 13 & 3 & 39 & $1,06 \%$ & $2,49 \%$ \\
\hline 12 & 5 & 60 & $1,77 \%$ & $3,83 \%$ \\
\hline 11 & 6 & 66 & $2,12 \%$ & $4,21 \%$ \\
\hline 10 & 9 & 90 & $3,18 \%$ & $5,74 \%$ \\
\hline 9 & 8 & 72 & $2,83 \%$ & $4,59 \%$ \\
\hline 8 & 16 & 128 & $5,65 \%$ & $8,17 \%$ \\
\hline 7 & 13 & 91 & $4,59 \%$ & $5,81 \%$ \\
\hline 6 & 12 & 72 & $4,24 \%$ & $4,59 \%$ \\
\hline 5 & 19 & 95 & $6,71 \%$ & $6,06 \%$ \\
\hline 4 & 24 & 96 & $8,48 \%$ & $6,13 \%$ \\
\hline 3 & 37 & 111 & $13,07 \%$ & $7,08 \%$ \\
\hline 2 & 36 & 72 & $12,72 \%$ & $4,59 \%$ \\
\hline 1 & 37 & 37 & $13,07 \%$ & $2,36 \%$ \\
\hline 0 & 32 & 0 & $11,31 \%$ & $0,00 \%$ \\
\hline Total: & 283 & 1567 & $100 \%$ & $100 \%$ \\
\hline
\end{tabular}

Tabla XI. Citas recibidas: artículos publicados en 2002.

\begin{tabular}{|c|c|c|c|c|}
\hline \multicolumn{5}{|c|}{ Articulos citados 2001} \\
\hline Citas/Art. & $N^{\circ}$ art. & $N^{\circ}$ citas & $\%$ Art. & $\%$ Citas \\
\hline 86 & 1 & 86 & $0,47 \%$ & $5,40 \%$ \\
\hline 38 & 1 & 38 & $0,47 \%$ & $2,39 \%$ \\
\hline 37 & 1 & 37 & $0,47 \%$ & $2,32 \%$ \\
\hline 33 & 1 & 33 & $0,47 \%$ & $2,07 \%$ \\
\hline 31 & 1 & 31 & $0,47 \%$ & $1,95 \%$ \\
\hline 29 & 2 & 58 & $0,94 \%$ & $3,64 \%$ \\
\hline 27 & 2 & 54 & $0,94 \%$ & $3,39 \%$ \\
\hline 25 & 1 & 25 & $0,47 \%$ & $1,57 \%$ \\
\hline 23 & 4 & 92 & $1,89 \%$ & $5,78 \%$ \\
\hline 22 & 2 & 44 & $0,94 \%$ & $2,76 \%$ \\
\hline 21 & 2 & 42 & $0,94 \%$ & $2,64 \%$ \\
\hline 20 & 2 & 40 & $0,94 \%$ & $2,51 \%$ \\
\hline 19 & 3 & 57 & $1,42 \%$ & $3,58 \%$ \\
\hline 18 & 2 & 36 & $0,94 \%$ & $2,26 \%$ \\
\hline 17 & 2 & 34 & $0,94 \%$ & $2,14 \%$ \\
\hline 16 & 2 & 32 & $0,94 \%$ & $2,01 \%$ \\
\hline 15 & 3 & 45 & $1,42 \%$ & $2,83 \%$ \\
\hline 14 & 2 & 28 & $0,94 \%$ & $1,76 \%$ \\
\hline 13 & 5 & 65 & $2,36 \%$ & $4,08 \%$ \\
\hline 12 & 5 & 60 & $2,36 \%$ & $3,77 \%$ \\
\hline 11 & 3 & 33 & $1,42 \%$ & $2,07 \%$ \\
\hline 10 & 8 & 80 & $3,77 \%$ & $5,03 \%$ \\
\hline 9 & 5 & 45 & $2,36 \%$ & $2,83 \%$ \\
\hline 8 & 2 & 16 & $0,94 \%$ & $1,01 \%$ \\
\hline 7 & 14 & 98 & $6,60 \%$ & $6,16 \%$ \\
\hline 6 & 13 & 78 & $6,13 \%$ & $4,90 \%$ \\
\hline 5 & 19 & 95 & $8,96 \%$ & $5,97 \%$ \\
\hline 4 & 22 & 88 & $10,38 \%$ & $5,53 \%$ \\
\hline 3 & 19 & 57 & $8,96 \%$ & $3,58 \%$ \\
\hline 2 & 21 & 42 & $9,91 \%$ & $2,64 \%$ \\
\hline 1 & 23 & 23 & $10,85 \%$ & $1,44 \%$ \\
\hline 0 & 19 & 0 & $8,96 \%$ & $0,00 \%$ \\
\hline Total: & 212 & 1592 & $100 \%$ & $100 \%$ \\
\hline
\end{tabular}

Tabla XII. Citas recibidas: artículos publicados en 2001.

\begin{tabular}{lrrrr}
\hline $\begin{array}{l}\text { Articulos citados } 2000 \\
\text { Citas/Articulo }\end{array}$ & $N^{0}$ articulos & $N^{\circ}$ citas & $\%$ Art. & \% Citas \\
\hline 58 & 1 & 58 & $0,52 \%$ & $3,92 \%$ \\
\hline 49 & 1 & 49 & $0,52 \%$ & $3,31 \%$ \\
\hline 33 & 2 & 66 & $1,04 \%$ & $4,46 \%$ \\
\hline 30 & 1 & 30 & $0,52 \%$ & $2,03 \%$ \\
\hline 29 & 2 & 58 & $1,04 \%$ & $3,92 \%$ \\
\hline 27 & 1 & 27 & $0,52 \%$ & $1,82 \%$ \\
\hline 26 & 1 & 26 & $0,52 \%$ & $1,76 \%$ \\
\hline 24 & 1 & 24 & $0,52 \%$ & $1,62 \%$ \\
\hline 23 & 1 & 23 & $0,52 \%$ & $1,55 \%$ \\
\hline 22 & 1 & 22 & $0,52 \%$ & $1,49 \%$ \\
\hline 20 & 2 & 40 & $1,04 \%$ & $2,70 \%$ \\
\hline 19 & 4 & 76 & $2,08 \%$ & $5,14 \%$ \\
\hline 18 & 3 & 54 & $1,56 \%$ & $3,65 \%$ \\
\hline 17 & 1 & 17 & $0,52 \%$ & $1,15 \%$ \\
\hline 16 & 4 & 64 & $2,08 \%$ & $4,32 \%$ \\
\hline 15 & 5 & 75 & $2,60 \%$ & $5,07 \%$ \\
\hline 14 & 5 & 70 & $2,60 \%$ & $4,73 \%$ \\
\hline 13 & 4 & 52 & $2,08 \%$ & $3,51 \%$ \\
\hline 12 & 6 & 72 & $3,13 \%$ & $4,86 \%$ \\
\hline 11 & 5 & 55 & $2,60 \%$ & $3,72 \%$ \\
\hline 10 & 7 & 70 & $3,65 \%$ & $4,73 \%$ \\
\hline 9 & 5 & 45 & $2,60 \%$ & $3,04 \%$ \\
\hline 8 & 9 & 72 & $4,69 \%$ & $4,86 \%$ \\
\hline 7 & 8 & 56 & $4,17 \%$ & $3,78 \%$ \\
\hline 6 & 12 & 72 & $6,25 \%$ & $4,86 \%$ \\
\hline 5 & 12 & 60 & $6,25 \%$ & $4,05 \%$ \\
\hline 4 & 11 & 44 & $5,73 \%$ & $2,97 \%$ \\
\hline 3 & 16 & 48 & $8,33 \%$ & $3,24 \%$ \\
\hline 2 & 18 & 36 & $9,38 \%$ & $2,43 \%$ \\
\hline 1 & 19 & 19 & $9,90 \%$ & $1,28 \%$ \\
\hline 0 & 24 & 0 & $12,50 \%$ & $0,00 \%$ \\
\hline Total: & 192 & 1480 & $100 \%$ & $100 \%$ \\
\hline & & & & \\
\hline & 1 & & &
\end{tabular}

Tabla XIII. Citas recibidas: artículos publicados en 2000.

\section{Conclusiones}

En este trabajo se realiza un análisis bibliométrico que permite tener una visión general de la actividad investigadora llevada a cabo en la UCLM desde el año 2000 al 2004, obtener un conocimiento mayor de la actividad desarrollada por el personal docente e investigador de la institución, así como de la calidad y repercusión de los trabajos medida a través de las citas bibliográficas que reciben.

Se observa un incremento progresivo en la cantidad de trabajos publicados desde el año 2000 (198) hasta el 2004 (346). El total de documentos estudiados es de 1394 ítems, de estos 1354 (el $97,1 \%$ del total) pertenecen al área de las ciencias, y están recogidos en el SCl. Suele tratarse de una investigación más básica, de interés para la comunidad científica internacional, que se difunde a través de publicaciones periódicas internacionales. Tanto las ciencias 
sociales (20 artículos) como el arte y las humanidades (20 artículos) tienen escasa representación internacional, posiblemente por tratarse de una investigación más local, o de alcance nacional, que afecta a un colectivo menor y difundida en publicaciones periódicas nacionales o regionales, no indizadas en el SSCI ni en el $\mathrm{A} \& \mathrm{HCl}$.

El inglés es el idioma dominante en todas las publicaciones $(97,6 \%)$ y es el elegido por el personal de la UCLM para difundir los resultados de sus investigaciones. En el caso de las ciencias entre las revistas más utilizadas no se encuentra ninguna nacional y en español sólo se escribe el $1 \%$ de todos los trabajos.

El análisis temático de la productividad de la UCLM indica que son: tecnología de los alimentos (89), química (analítica (87), orgánica (79), física (78) e inorgánica y nuclear (77)) con un total de 410 artículos las materias de las que más trabajos se han publicado. $Y$ energía y combustibles, espectroscopia, estadística y probabilidad, farmacología y farmacia, genética, informática e ingeniería medioambiental, con 10 trabajos publicados en cada una de las categorías las materias con menos actividad investigadora con repercusión internacional.

Durante los cinco años estudiados las materias que han recibido una mayor cantidad de citas en ciencias son: química orgánica (670), química inorgánica y nuclear (556), bioquímica y biología molecular (479), química física (438) y neurociencias (402). En el año 2001 se publican los trabajos que más se han citado en trabajos posteriores y en 2004 el que menos. Y las materias que menos citas han recibido son: estadística y probabilidad (14), informática-aplicaciones (25), ingeniería metalúrgica (25) e investigaciones operativas (27). Biología celular $(12,38)$, electroquímica (11) y óptica (11) son las materias en las que la ratio citas por artículo alcanza los valores más altos.

La visibilidad internacional de las publicaciones, se ha considerado a partir del FI y el JCR para cada categoría temática del $\mathrm{SCl}$. Todos los trabajos se publicaron en 620 títulos de revistas y en 21 se difundieron el $21 \%$ de los artículos (285). De las 21 publicaciones preferidas por los investigadores de la UCLM para difundir los resultados de sus investigaciones, 13 se sitúan en el primer cuartil de su categoría, 6 en el segundo, 1 en el tercero y 1 en el cuarto. Por lo que estos trabajos se han difundido en títulos de visibilidad internacional.

Centrándose en las revistas científicas que han publicado los trabajos que han obtenido mayor número de citas en trabajos posteriores, 36 publicaciones $(5,69 \%)$ han publicado 51 artículos $(3,76 \%)$ que han recibido 1.417 citas (el 21,67\%). 27 están en el primer cuartil de su categoría, 7 en el segundo, y respectivamente una en el tercer y cuarto cuartil. Los artículos más citados se han publicado en revistas con alta visibilidad internacional.

Los 1.354 artículos recogidos en la base de datos del $\mathrm{SCl}$ han recibido 6.537 citas. El $78,95 \%$ de los analizados se han citado en alguna ocasión. En el año 2001 se publicó el único documento que ha superado las 80 citas (86), es decir el más citado de los últimos 6 años. Durante el año 2003 se publicaron los artículos que más se citaron (23,95\%).

Se observa que si bien anualmente aumenta la producción de trabajos, la citación de los mismos desciende, lo que implica un descenso en la calidad de los mismos, o una falta de interés de los temas tratados por parte de la comunidad científica.

Los investigadores saben donde publicar sus artículos si quieren obtener una visibilidad internacional, de modo que los artículos más citados han sido publicados en su mayoría por revistas con factor de impacto, recogidas en el JCR, en el primer cuartil de su categoría.

\section{Referencias}

Plan Regional de Investigación Científica, Desarrollo Tecnológico e Innovación (PRINCET). http://www.jccm.es/ educacion/ciencia/princet.htm (2006-05-02).

Plan Regional de Investigación Científica y Desarrollo Tecnológico PRICYT: 2000 - 2003. Toledo: JCCM, 2000.

Bordons, M.; Gómez, I (1997). La actividad científica española a través de indicadores bibliométricos en el período 1990-93. // Revista general de información y documentación. 7:2 (1997) 69-86.

Fernández, M. T.; Cabrero, A.; Zulueta, M.A.; Gómez, I. (1993). Constructing a relational database for bibliometric analysis. // Research evaluation. 3:1 (1993) 55-62.

Fernández, T.; Bordons, M.; Sancho, R.; Gómez, I. (1999). Difusión internacional de la investigación científica española en ciencia y tecnología en el período 1991-1996. // Arbor. 162: 639 (1999) 327-45.

Ferreiro, L. (1993). Bibliometría: análisis bivariante. Madrid: Eypasa, 1993.

Gómez, I.; Fernández, M. T,; Bordons, M.; Morillo, F. (2003). Región de Murcia: análisis de la producción científica, estudio bibliométrico del período 1998-2000. Murcia: Fundación Séneca, 2003.

Gómez, I.; Bordons, M. (1996). Limitaciones en el uso de los indicadores bibliométricos para la evaluación científica. // Política científica. 46 (1996) 21-26.

Licea de Arenas, J.; Rodríguez, J.V.; Gómez, J.A.; Arenas, M. (2005). Comunicación de conocimiento. ¿Habilidad de los profesores universitarios?. // Information research. 11:1 (2005). http://informationr.net/ir/11paper243.html

Martín, M. J.; Rey, J.; Plaza, L. M. (1999). La investigación española en revistas nacionales de ciencia y tecnología 
durante el período 1991-1996: estudio bibliométrico. // Arbor. 162:639 (1999) 307-25.

Moya, F.; Chinchilla, Z.; Corera, E.; Vargas, J.; Muñoz, F.; Herrero, V. (2005). Análisis de dominio institucional: la producción científica de la universidad de Granada $(\mathrm{SCl}$ 1991-99). // Revista española de documentación científica. 28:2 (2005) 170-195.

Muñoz, E. (2003). 20 Años de ciencia en la región de Murcia: análisis bibliométrico. Murcia: Academia de ciencias de la Región de Murcia, 2003.
Pérez, J. R. (1999). La ciencia y la tecnología españolas a través de sus publicaciones. // Arbor. 162:639 (1999) 303-6.

Sanz, E.; Martín, C.; García, C.; Suárez, C.; Lascurain, M.L. (2002). La actividad científica española en ciencias médicas en el período 1991-1999 [en línea]. // Acimed 10:1 (2002) 1-2. http://scielo.sld.cu/scielo.php?Script=sci arttext\&pid=S102494352002000100002\&lng=es\&nrm=iso

\section{Apéndice}

\begin{tabular}{lrrrr}
\hline Materias & $N^{\circ}$ Art. & $\%$ art. & $N^{\circ}$ citas & Citas/Art. \\
\hline Tecnología de los alimentos & 89 & $6,6 \%$ & 355 & 3,99 \\
\hline Química analítica & 87 & $6,4 \%$ & 385 & 4,43 \\
\hline Química orgánica & 79 & $5,8 \%$ & 670 & 8,48 \\
\hline Química física & 78 & $5,8 \%$ & 438 & 5,62 \\
\hline Química inorgánica y nuclear & 77 & $5,7 \%$ & 556 & 7,22 \\
\hline Ciencias medioambientales & 67 & $4,9 \%$ & 337 & 5,03 \\
\hline Ecología & 58 & $4,3 \%$ & 269 & 4,64 \\
\hline Matemáticas aplicadas & 58 & $4,3 \%$ & 142 & 2,45 \\
\hline Neurociencias & 58 & $4,3 \%$ & 402 & 6,93 \\
\hline Ingeniería eléctrica y electrónica & 53 & $3,9 \%$ & 308 & 5,81 \\
\hline Bioquímica y biología molecular & 52 & $3,8 \%$ & 479 & 9,21 \\
\hline Ingeniería química & 47 & $3,5 \%$ & 211 & 4,49 \\
\hline Química aplicada & 45 & $3,3 \%$ & 213 & 4,73 \\
\hline Zoología & 41 & $3,0 \%$ & 133 & 3,24 \\
\hline Bioquímica, métodos e investigación & 36 & $2,7 \%$ & 176 & 4,89 \\
\hline Ciencia de los materiales & 32 & $2,4 \%$ & 140 & 4,38 \\
\hline Química & 32 & $2,4 \%$ & 171 & 5,34 \\
\hline Física de fluidos & 31 & $2,3 \%$ & 193 & 6,23 \\
\hline Informática, inteligencia artificial & 31 & $2,3 \%$ & 70 & 2,26 \\
\hline Recursos hídricos & 14 & $2,3 \%$ & 143 & 4,61 \\
\hline Física nuclear, molecular y química & 29 & $2,1 \%$ & 282 & 9,72 \\
\hline Física matemática & 13 & $1,0 \%$ & 161 & 12,38 \\
\hline Informática, ingeniería del software & 26 & $1,9 \%$ & 143 & 5,5 \\
\hline Meteorología & 25 & $1,8 \%$ & 50 & 2 \\
\hline Física aplicada & 25 & $1,8 \%$ & 118 & 4,72 \\
\hline Física, condensación de materias & 24 & $1,8 \%$ & 160 & 6,67 \\
\hline Agricultura & 24 & $1,8 \%$ & 65 & 2,71 \\
\hline Matemáticas & 22 & $1,6 \%$ & 124 & 5,64 \\
\hline Veterinaria & 22 & $1,6 \%$ & 43 & 1,95 \\
\hline Biotecnología y microbiología aplicada & 21 & $1,6 \%$ & 95 & 4,52 \\
\hline Horticultura & 19 & $1,4 \%$ & 50 & 2,63 \\
\hline Física & 19 & $1,4 \%$ & 61 & 3,21 \\
\hline Geociencias & 18 & $1,3 \%$ & 159 & 8,83 \\
\hline Agronomía & 18 & $1,3 \%$ & 77 & 4,28 \\
\hline Biodiversidad, Conservación & 17 & $1,3 \%$ & 67 & 3,94 \\
\hline Ingeniería mecánica & 16 & $1,2 \%$ & 61 & 3,81 \\
\hline Ingeniería metalúrgica & 16 & $1,2 \%$ & 41 & 2,56 \\
\hline Investigación operativa & $1,2 \%$ & 25 & 1,56 \\
\hline Mecánica & $1,2 \%$ & 27 & 1,69 \\
\hline Ingeniería de montes & 50 & 3,13 \\
\hline Microbiología & 67 & 4,47 \\
\hline Biofíca & $13 \%$ & 132 & 9,43 \\
\hline
\end{tabular}




\begin{tabular}{lrrrr}
\hline Materias & $N^{\circ}$ Art. & $\%$ art. & $N^{\circ}$ citas & Citas/Art. \\
\hline Electroquímica & 13 & $1,0 \%$ & 143 & 11 \\
\hline Endocrinología y metabolismo & 13 & $1,0 \%$ & 128 & 9,85 \\
\hline Informática, teoría y métodos & 13 & $1,0 \%$ & 36 & 2,77 \\
\hline Parasitología & 12 & $0,9 \%$ & 34 & 2,83 \\
\hline Energía y combustibles & 10 & $0,7 \%$ & 35 & 3,5 \\
\hline Espectroscopia & 10 & $0,7 \%$ & 48 & 4,8 \\
\hline Estadística y probabilidad & 10 & $0,7 \%$ & 14 & 1,4 \\
\hline Farmacología y farmacia & 10 & $0,7 \%$ & 50 & 5 \\
\hline Genética & 10 & $0,7 \%$ & 69 & 6,9 \\
\hline Informática, aplicaciones & 10 & $0,7 \%$ & 25 & 2,5 \\
\hline Ingeniería medioambiental & 10 & $0,7 \%$ & 86 & 8,6 \\
\hline Total: & 1663 & & 8762 & \\
\hline
\end{tabular}

Tabla III. Distribución por materias de los artículos.

\begin{tabular}{|c|c|c|c|c|c|c|c|c|c|c|}
\hline Materias & $\begin{array}{l}\text { art } \\
00\end{array}$ & $\begin{array}{c}\text { citas } \\
00\end{array}$ & $\begin{array}{l}\text { art } \\
01\end{array}$ & $\begin{array}{c}\text { citas } \\
01\end{array}$ & $\begin{array}{l}\text { art } \\
02\end{array}$ & $\begin{array}{c}\text { citas } \\
02\end{array}$ & $\begin{array}{l}\text { art } \\
03\end{array}$ & $\begin{array}{c}\text { citas } \\
03\end{array}$ & $\begin{array}{l}\text { art } \\
04\end{array}$ & $\begin{array}{c}\text { citas } \\
04\end{array}$ \\
\hline Agricultura & 4 & 32 & 2 & 15 & 6 & 46 & 6 & 20 & 4 & 11 \\
\hline Agronomía & 3 & 35 & 2 & 17 & 1 & 5 & 5 & 8 & 6 & 2 \\
\hline Biodiversidad, Conservación & 1 & 4 & 3 & 24 & 1 & 4 & 4 & 17 & 7 & 12 \\
\hline Biofísica & 1 & 3 & 3 & 65 & 5 & 46 & 4 & 18 & 1 & 0 \\
\hline Biología celular & 2 & 11 & 3 & 95 & 5 & 38 & 1 & 5 & 2 & 12 \\
\hline Bioquímica y biología molecular & 6 & 94 & 8 & 174 & 14 & 104 & 16 & 55 & 8 & 52 \\
\hline Bioquímica, métodos e investigación & 3 & 21 & 4 & 25 & 15 & 67 & 10 & 27 & 4 & 36 \\
\hline Biotecnología y microbiología aplicada & 1 & 7 & 1 & 4 & 2 & 5 & 6 & 15 & 9 & 19 \\
\hline Ciencia de los materiales & 2 & 12 & 8 & 33 & 13 & 69 & 5 & 14 & 4 & 12 \\
\hline Ciencias medioambientales & 13 & 83 & 10 & 102 & 8 & 56 & 17 & 71 & 19 & 25 \\
\hline Ecología & 1 & 4 & 11 & 120 & 10 & 52 & 16 & 53 & 20 & 40 \\
\hline Electroquímica & 0 & 0 & 2 & 27 & 4 & 32 & 2 & 52 & 5 & 32 \\
\hline Endocrinología y metabolismo & 1 & 8 & 3 & 33 & 3 & 37 & 4 & 29 & 2 & 21 \\
\hline Energía y combustibles & 1 & 11 & 1 & 12 & 2 & 5 & 3 & 5 & 3 & 2 \\
\hline Espectroscopia & 4 & 31 & 0 & 0 & 1 & 7 & 1 & 7 & 4 & 3 \\
\hline Estadística y probabilidad & 0 & 0 & 4 & 11 & 2 & 0 & 0 & 0 & 4 & 3 \\
\hline Farmacología y farmacia & 5 & 23 & 0 & 0 & 2 & 13 & 2 & 10 & 1 & 4 \\
\hline Física & 2 & 81 & 3 & 20 & 4 & 10 & 3 & 4 & 6 & 44 \\
\hline Física aplicada & 7 & 97 & 2 & 5 & 8 & 28 & 4 & 11 & 3 & 19 \\
\hline Física de fluidos & 2 & 34 & 5 & 50 & 6 & 45 & 11 & 59 & 7 & 5 \\
\hline Física matemática & 1 & 27 & 2 & 21 & 8 & 32 & 9 & 38 & 6 & 25 \\
\hline Física nuclear, molecular y química & 8 & 121 & 3 & 66 & 5 & 40 & 8 & 33 & 5 & 22 \\
\hline Física, condensación de materias & 0 & 0 & 6 & 29 & 7 & 18 & 4 & 6 & 7 & 12 \\
\hline Genética & 3 & 11 & 1 & 31 & 2 & 13 & 3 & 5 & 1 & 9 \\
\hline Geociencias & 3 & 14 & 4 & 25 & 4 & 17 & 4 & 16 & 3 & 5 \\
\hline Horticultura (Plant Sciences) & 2 & 5 & 3 & 11 & 4 & 19 & 6 & 16 & 4 & 10 \\
\hline Informática, aplicaciones & 4 & 12 & 0 & 0 & 2 & 4 & 2 & 7 & 2 & 2 \\
\hline Informática, ingeniería del software & 3 & 7 & 7 & 21 & 3 & 7 & 9 & 14 & 3 & 1 \\
\hline Informática, inteligencia artificial & 2 & 1 & 8 & 20 & 7 & 15 & 8 & 30 & 6 & 4 \\
\hline Informática, teoría y métodos & 2 & 2 & 3 & 14 & 1 & 1 & 4 & 12 & 3 & 7 \\
\hline Ingeniería mecánica & 4 & 20 & 0 & 0 & 3 & 3 & 4 & 15 & 5 & 3 \\
\hline Ingeniería de montes & 2 & 12 & 3 & 29 & 3 & 17 & 4 & 5 & 3 & 4 \\
\hline Ingeniería eléctrica y electrónica & 8 & 80 & 7 & 43 & 16 & 125 & 8 & 45 & 14 & 15 \\
\hline Ingeniería medioambiental & 3 & 14 & 2 & 40 & 2 & 15 & 2 & 15 & 1 & 2 \\
\hline
\end{tabular}




\begin{tabular}{|c|c|c|c|c|c|c|c|c|c|c|}
\hline Materias & $\begin{array}{l}\text { art } \\
00\end{array}$ & $\begin{array}{c}\text { citas } \\
00\end{array}$ & $\begin{array}{l}\text { art } \\
01\end{array}$ & $\begin{array}{c}\text { citas } \\
01\end{array}$ & $\begin{array}{l}\text { art } \\
02 \\
\end{array}$ & $\begin{array}{c}\text { citas } \\
02\end{array}$ & $\begin{array}{l}\text { art } \\
03\end{array}$ & $\begin{array}{c}\text { citas } \\
03\end{array}$ & $\begin{array}{l}\text { art } \\
04 \\
\end{array}$ & $\begin{array}{c}\text { citas } \\
04\end{array}$ \\
\hline Ingeniería metalúrgica & 2 & 0 & 2 & 2 & 4 & 10 & 3 & 10 & 5 & 3 \\
\hline Ingeniería química & 7 & 18 & 5 & 34 & 13 & 76 & 9 & 34 & 13 & 49 \\
\hline Investigación operativa & 1 & 1 & 0 & 0 & 4 & 8 & 7 & 13 & 4 & 5 \\
\hline Matemáticas & 4 & 7 & 6 & 23 & 3 & 7 & 7 & 6 & 2 & 0 \\
\hline Matemáticas aplicadas & 4 & 9 & 15 & 60 & 11 & 22 & 17 & 17 & 11 & 34 \\
\hline Mecánica & 6 & 38 & 3 & 1 & 2 & 7 & 1 & 2 & 4 & 2 \\
\hline Meteorología & 2 & 17 & 4 & 35 & 2 & 14 & 10 & 46 & 7 & 6 \\
\hline Microbiología & 3 & 42 & 2 & 6 & 3 & 6 & 4 & 23 & 3 & 14 \\
\hline Neurociencias & 4 & 32 & 8 & 89 & 13 & 148 & 17 & 89 & 16 & 44 \\
\hline Óptica & 4 & 38 & 7 & 99 & 1 & 7 & 1 & 5 & 1 & 5 \\
\hline Parasitología & 0 & 0 & 1 & 5 & 1 & 4 & 3 & 12 & 7 & 13 \\
\hline Química & 6 & 40 & 4 & 29 & 6 & 28 & 6 & 48 & 10 & 26 \\
\hline Química analítica & 11 & 102 & 12 & 55 & 27 & 94 & 18 & 57 & 19 & 77 \\
\hline Química aplicada & 5 & 33 & 7 & 57 & 11 & 59 & 10 & 35 & 12 & 29 \\
\hline Química física & 16 & 141 & 17 & 102 & 17 & 117 & 13 & 57 & 15 & 21 \\
\hline Química inorgánica y nuclear & 18 & 159 & 12 & 126 & 13 & 117 & 16 & 93 & 18 & 61 \\
\hline Química orgánica & 17 & 181 & 19 & 219 & 15 & 133 & 13 & 92 & 15 & 45 \\
\hline Recursos hídricos & 5 & 36 & 5 & 64 & 3 & 19 & 7 & 17 & 11 & 7 \\
\hline Tecnología de los alimentos & 15 & 103 & 12 & 57 & 13 & 68 & 27 & 96 & 22 & 31 \\
\hline Veterinaria & 1 & 13 & 3 & 8 & 5 & 32 & 6 & 22 & 6 & 20 \\
\hline Zoología & 4 & 33 & 2 & 9 & 7 & 25 & 12 & 39 & 16 & 27 \\
\hline Total: & 239 & 1960 & 271 & 2262 & 355 & 1996 & 405 & 1550 & 403 & 994 \\
\hline
\end{tabular}

Tabla IV. Distribución por materias de los artículos generados y citas recibidas para cada año.

\begin{tabular}{llllrl}
\hline Titulo & & & & & \\
\hline J. Org. Chem. & 4 & 94 & 3,462 & $7 / / 58$ & 1 \\
\hline IEEE Trans. Power Syst. & 3 & 86 & 0,821 & $84 / / 209$ & 2 \\
\hline J. Acoust. Soc. Am. & 3 & 73 & 1,482 & $5 / / 26$ & 1 \\
\hline Organometallics & 3 & 71 & 3,196 & $8 / / 45$ & 1 \\
\hline BBAProtein Struct. Molec. Enzym. & 2 & 53 & 3,079 & $95 / / 261$ & 2 \\
\hline Eur. J. Neurosci. & 2 & 46 & 3,820 & $42 / / 198$ & 1 \\
\hline J. Electrochem. Soc. & 2 & 51 & 2,356 & $5 / / 20$ & 1 \\
\hline Phys. Rev. A & 2 & 64 & 2,902 & $7 / / 54$ & 1 \\
\hline Phys. Rev. Lett. & 2 & 81 & 7,218 & $4 / / 67$ & 1 \\
\hline Tetrahedron Lett. & 2 & 46 & 2,484 & $15 / / 58$ & 1 \\
\hline Appl. Phys. Lett. & 1 & 49 & 4,308 & $4 / / 79$ & 1 \\
\hline Biochem. J. & 1 & 30 & 4,278 & $57 / / 261$ & 1 \\
\hline Biochemistry & 1 & 33 & 4,008 & $65 / / 261$ & 1 \\
\hline Biosystems & 1 & 26 & 1,016 & $34 / / 64$ & 3 \\
\hline Brain Res. Bull. & 1 & 24 & 2,429 & $89 / / 198$ & 2 \\
\hline Cereb. Cortex & 1 & 25 & 5,322 & $19 / 198$ & 1 \\
\hline Chem. Phys. Lett. & 1 & 20 & 2,438 & $29 / / 108$ & 2 \\
\hline Embo J. & 1 & 86 & 10,492 & $13 / / 261$ & 1 \\
\hline Endocrinology & 1 & 24 & 5,151 & $14 / / 87$ & 1 \\
\hline Inorg. Chem. & 1 & 23 & 3,454 & $6 / / 45$ & 1 \\
\hline Int. J. Food Microbiol. & 1 & 20 & 2,490 & $4 / / 94$ & 1 \\
\hline & & & & &
\end{tabular}




\begin{tabular}{lrrrrr}
\hline Titulo & & & & & \\
\hline J. Appl. Electrochem. & 1 & 22 & 0,982 & $16 / / 20$ & 4 \\
\hline J. Chromatogr. A & 1 & 20 & 3,359 & $12 / / 51$ & 1 \\
\hline J. Evol. Biol. & 1 & 31 & 2,893 & $21 / / 107$ & 1 \\
\hline J. Mater. Chem. & 1 & 35 & 2,721 & $25 / / 108$ & 1 \\
\hline J. Neurosci. & 1 & 25 & 7,907 & $12 / / 198$ & 1 \\
\hline J. Organomet. Chem. & 1 & 22 & 1,905 & $17 / / 45$ & 2 \\
\hline J. Phys. Chem. A & 1 & 25 & 2,639 & $27 / / 108$ & 1 \\
\hline Dalton Trans. & 1 & 29 & 2,926 & $9 / / 45$ & 1 \\
\hline J. Urol. & 1 & 24 & 3,713 & $6 / / 52$ & 1 \\
\hline Neuroscience & 1 & 21 & 3,456 & $51 / / 198$ & 2 \\
\hline Photogramm. Eng. Remote Sens. & 1 & 22 & 1,574 & $9 / / 29$ & 2 \\
\hline Physica D & 1 & 22 & 1,666 & $6 / / 162$ & 1 \\
\hline Phys. Rev. E & 1 & 27 & 2,352 & $3 / / 21$ & 1 \\
\hline Proc. Natl. Acad. Sci. USA & 1 & 29 & 10,452 & $3 / / 45$ & 1 \\
\hline Water Res. & 1 & 38 & 2,304 & $3 / / 35$ & 1 \\
\hline Total & 51 & 1417 & & & \\
\hline
\end{tabular}

Tabla VI. Revistas que publicaron los artículos más citados de acuerdo con el SCI. 